\title{
Zipping dielectric elastomer actuators: characterization, design and modeling
}

Maffli, Luc; Rosset, Samuel; Shea, Herbert

- $\quad$ Published in: Smart Materials and Structures (ISSN: 1361-665X), vol. 22, num. 10, p. 104013

- $\quad$ Bristol: Iop Publishing Ltd, 2013

\begin{abstract}
We report on miniature dielectric elastomer actuators (DEAs) operating in zipping mode with an analytical model that predicts their behavior. Electrostatic zipping is a well-known mechanism in silicon MEMS to obtain large deformations and forces at lower voltages than for parallel plate electrostatic actuation. We extend this concept to DEAs, which allows us to obtain much larger out-ofplane displacements compared to silicon thanks to the softness of the elastomer membrane. We study experimentally the effect of sidewall angles and elastomer prestretch on $2.3 \mathrm{~mm}$ diameter actuators with PDMS membranes. With $15^{\circ}$ and $22.5^{\circ}$ sidewall angles, the devices zip in a bistable manner down $300 \mu \mathrm{m}$ to the bottom of the chambers. The highly tunable bistable behavior is controllable by both chamber geometry and membrane parameters. Other specific characteristics of zipping DEAs include well-controlled deflected shape, tunable displacement versus voltage characteristics to virtually any shape, including multi-stable modes, sealing of embedded holes or channels for valving action and the reduction of the operating voltage. These properties make zipping DEAs an excellent candidate for applications such as integrated microfluidics actuators or Braille displays.
\end{abstract}

\section{Reference}

- $\quad$ EPFL-ARTICLE-188614

- $\quad$ doi:10.1088/0964-1726/22/10/104013

(C) 2013 IOP publishing Ltd 


\title{
Zipping Dielectric Elastomer Actuators: characterization, design and modeling
}

\author{
L. Maffli, S. Rosset and H. R. Shea \\ Microsystems for Space Technologies Laboratory (LMTS), Ecole Polytechnique Fédérale de \\ Lausanne (EPFL), Neuchâtel, Switzerland \\ E-mail: luc.maffli@epfl.ch
}

\begin{abstract}
We report on miniature Dielectric Elastomer Actuators (DEAs) operating in zipping mode with an analytical model that predicts their behavior. Electrostatic zipping is a well-known mechanism in silicon MEMS to obtain large deformations and forces at lower voltages than for parallel plate electrostatic actuation. We extend this concept to DEAs, which allows us to obtain much larger out-of-plane displacements compared to silicon thanks to the softness of the elastomer membrane. We study experimentally the effect of sidewalls angle and elastomer prestretch on 2.3 $m m$ diameter actuators with PDMS membranes. With $15^{\circ}$ and $22.5^{\circ}$ sidewalls angle, the devices zip in a bistable manner down $300 \mu m$ to the bottom of the chambers. The highly tunable bistable behavior is controllable by both chamber geometry and membrane parameters. Other specific characteristics of zipping DEAs include well-controlled deflected shape, tunable displacement vs. voltage characteristic to virtually any shape including multi-stable modes, sealing of embedded holes or channels for valving action and reduction of the operating voltage. These properties make zipping DEAs an excellent candidate for applications like integrated microfluidics actuators or Braille displays.
\end{abstract}

Keywords: EAP, zipping dielectric elastomer actuators, DEA, silicone, microfluidics, MEMS.

\section{Introduction}

Dielectric Elastomer Actuators (DEAs), also called artificial muscles, have unmatched combination of large actuation strains (typically $25 \%$ linear strain for long-lifetime operation, but up to $217 \%$ has been reported with silicone elastomers [1], recently up to $1692 \%$ area strain voltage-triggered deformation for an acrylic membrane [2]) and energy densities as high as $0.75 \mathrm{MJ} / \mathrm{m}^{3}$ [3]. They are only starting to appear in mass produced devices, and could revolutionize many areas of application [4]: soft robotics [5], haptic feedback [6], tunable optics [7], loudspeakers [8], cell mechanical stimulation [9], energy harvesting [10,11], smart coupled sensing and actuation units [12].

One of the areas that would greatly benefit from DEAs is microfluidics, in particular, largescale integrated microfluidic (MLSI) chips. MLSI consists of arrays of independently-controlled pneumatic actuators, which produce both a pumping and valving action $[13,14]$. These simple and robust actuator units consists of a soft silicone membrane that is deflected by a pneumatic pressure to close or open a channel or a chamber with embedded channels (figure 1) [15]. However, despite the small size of the chips, these pneumatically-activated systems are not portable as they require 

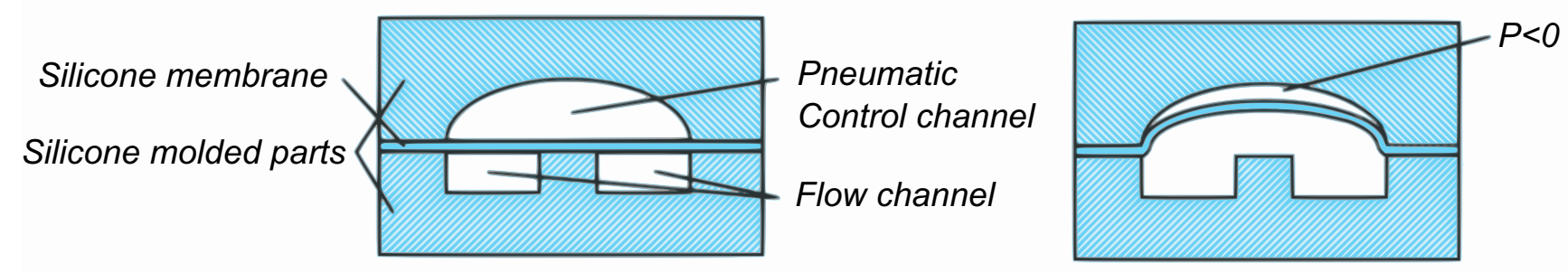

Figure 1. Commonly-used pneumatic normally-off microfluidic valve $[14,15]$

extensive off-chip equipment, such as a pressure or vacuum source, liquid pumps and a large set of pneumatic valves to selectively activate each chamber: the small chip remains bound in the lab. But having truly portable devices is required for point-of-care and home use applications, which will probably be the commercial breakthrough of lab-on-chips. Replacing the pneumatic actuators by electrical actuation would greatly reduce the size of the off-chip components, as for instance proposed by Chang et al, who developed small electrostatically active chambers [16].

DEAs could be the ideal candidates to replace the pneumatic-powered valves by electricallyactuated ones, since both operate by deflecting soft elastomer membranes. DEAs consist of a soft elastomer membrane sandwiched between two compliant electrodes, generally supported by a frame. As a high electric field is applied across the elastomer, it compresses in thickness and expands in-plane due to the incompressibility condition, resulting in very large strains. Since its introduction by Pelrine et al. in 1998 [17], most of the efforts of the scientific community have been focused on this successful DEA configuration. To achieve out-of plane motion, frames with joints and hinges [18,19], minimum energy structures [20], buckling-mode devices [21] or the use of offset pressures [22] have been reported. Another broadly studied way to achieve out-of-plane actuation is to use the thickness compression of stacked or folded layers [23, 24].

Making an actuator for microfluidics is a challenge as one requires: well-controlled out-ofplane deflected shape, liquid-tight sealing of chamber sidewalls, low voltage operation, and bistable operation. There is a gap to bridge between the state-of-the art DEA and these requirements for exerting a force on conductive liquids. We propose zipping DEAs as a variant that precisely meets the requirements for replacing the pneumatic microfluidic valves.

\section{Zipping DEAs}

In this work, we study theoretically and experimentally a novel DEA structure called zipping DEA, which moves away from the traditional DEA sandwich structure, using a compliant and a rigid electrode. It offers a new set of characteristics including well-controlled deflected shape, sealing ability, and tunable deflection versus voltage profile to virtually any behavior including multi-stable modes.

The zipping mechanism is well-known in silicon MEMS since the 90's [25] and has been used in a variety of devices, from tilting mirrors to microrelays. The Zipping actuators allows reaching both high forces and large stable displacements at low voltages thanks to the use of a few $\mu m$ thick high-quality rigid dielectric onto which a conducting flexure (usually silicon) is unrolled or zipped. 

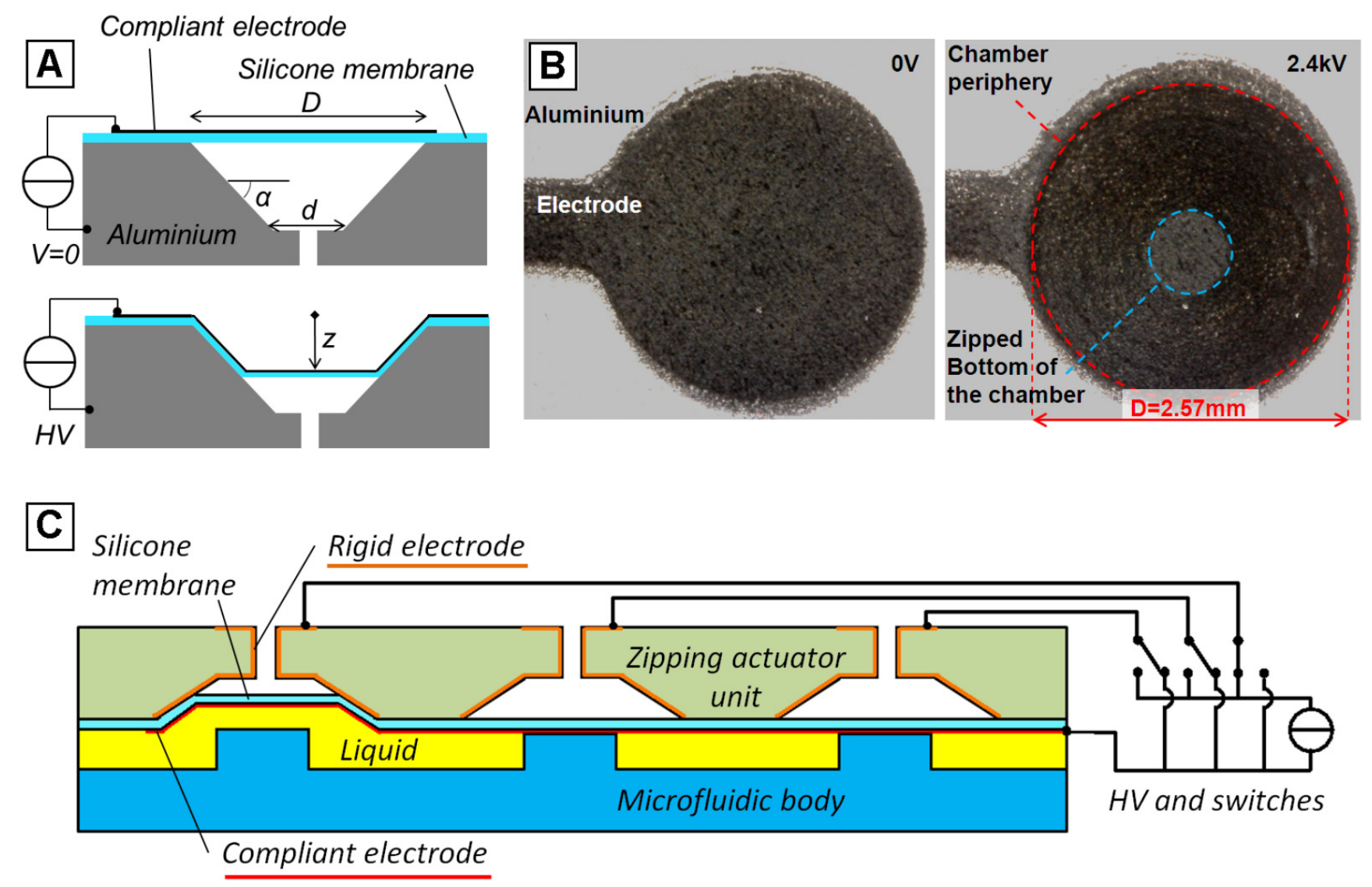

Figure 2. A Schematic cross section of a our conical-shape zipping actuator. The three essential geometrical parameters are the top and bottom diameters $D$ and $d$ and the sidewall angle $\alpha$. As a voltage is applied between the compliant electrode and the conductive chamber body, the membrane zips down in the chamber. B Photograph (top view) of an unactuated (left) and actuated (right) zipping actuator. Sidewalls angle $\alpha=15^{\circ}$, membrane thickness $23.5 \mu \mathrm{m}$, prestretch 1.32, geometrically-constrained zipping depth $z=280 \mu \mathrm{m}$. C Cross-section along the channel of a proposed peristaltic zipping micropump composed of three actuators. In this configuration, no electric field is applied in the liquid.

We extend this actuation mode to DEAs. Unlike the conventional DEA actuation mechanism, the elastomer membrane of a zipping DEA is not squeezed by the electrostatic force between two compliant electrodes, but a single soft electrode is attracted toward a fixed one (figure 2A). For an alternate zipping geometry modeled but not studied experimentally in this work, the operating voltage can be significantly reduced using a thin high quality rigid dielectric [26,27].

Our devices consist of an elastomer membrane bonded to a conical cavity. Our conical chambers have a top diameter of $2.3 \mathrm{~mm}$ and are milled in an aluminum plate, which acts as rigid electrode. A hole is bored at the bottom of the chamber to let the air out during actuation. A $25 \mu \mathrm{m}$ thick pre-stretched silicone membrane with a silicone-carbon black compliant electrode patterned on top is bonded over the chambers. The suspended membrane is flat in non-actuated state. As the voltage between the compliant electrode and the rigid Al electrode increases, so does the electric field across the elastomer membrane. Since the electric field is inversely proportional to the gap between the electrodes and due to the sloped sidewalls, the largest force is present at 
the periphery of the membrane, where the motion starts. The electrostatic force pulls down the membrane like a zipper, generating large out-of-plane deformation. Above a threshold voltage, the energetically most stable position is with a membrane completely deflected: the membrane jumps down to the bottom of the chamber (figure 2B).

The figure $2 \mathrm{C}$ represents a peristaltic zipping micropump based on three normally-off actuators. As the voltage is turned on, the membrane zips inside the cavity, pumping the liquid inside the chamber and opening the valve. Compared to the other pump structure we presented in [27], this flipped actuator design keeps the liquid free from the electric field. This allows handling fluids regardless of their conductivity, and prevents affecting biological samples such as cells.

\section{Modeling of the zipping actuators}

The most common approach to model the static displacement of electrostatic actuators as a function of the voltage and the initial conditions involves computing the energy contributions to find the energy minima. Like when modeling conventional DEAs, one has to introduce the hyperelastic stiffening of the material. Unlike for most DEA modeling, the membrane thickness and stretch state cannot be considered as constant over the actuator, since it is in hard contact with the sidewalls. We built a model which outputs the vertical deflection $z$ in function of the voltage, taking as parameters the chamber geometry, the elastomer properties (electrical and hyperelastic energy density) and the surface roughness of the rigid electrode, with the goal of designing actuators whose membrane can be zipped down to the bottom of the cavity without reaching the dielectric breakdown of the elastomer.

Saif made a detailed analytical study of the behavior of a zipping cavity to be used as a pump [28]. However, his model is not directly applicable to zipping EAPs because of the assumptions linked to the type of materials assumed in his model ( $5 \mu \mathrm{m}$ thick polyimide membrane). DEA membranes are between 3 to 4 orders of magnitude softer and work in a hyperelastic stress-strain domain, but also the contact between the rubbery elastomer membrane and the rigid electrode tends toward the hypothesis of non-slipping conditions, which are not used with Saifs low-friction membrane materials.

We model two fundamentally different zipping structures. In the first structure (figure 3A), the electric field is applied across the elastomer membrane. It can be seen as a stack compliant electrode - elastomer membrane - rigid electrode. The second one is similar to the silicon MEMS zipping devices: the compliant electrode is patterned on the membrane backside and is pulled in direct contact with a thin high-quality rigid dielectric coating that covers the rigid electrode (figure 3B). It could be described as a stack of elastomer membrane - compliant electrode - rigid dielectric - rigid electrode. The electric field builds up in the thin rigid dielectric, hence needing less voltage to reach the same amount of electrostatic energy. It also means that the mechanical and electrostatic optimization of the device are decoupled between the membrane and the rigid dielectric. As a direct consequence, the actuation is no more limited by the breakdown field of the membrane but by the rigid dielectric. This family of zipping devices were studied experimentally by Gebbers et al. [26]. 


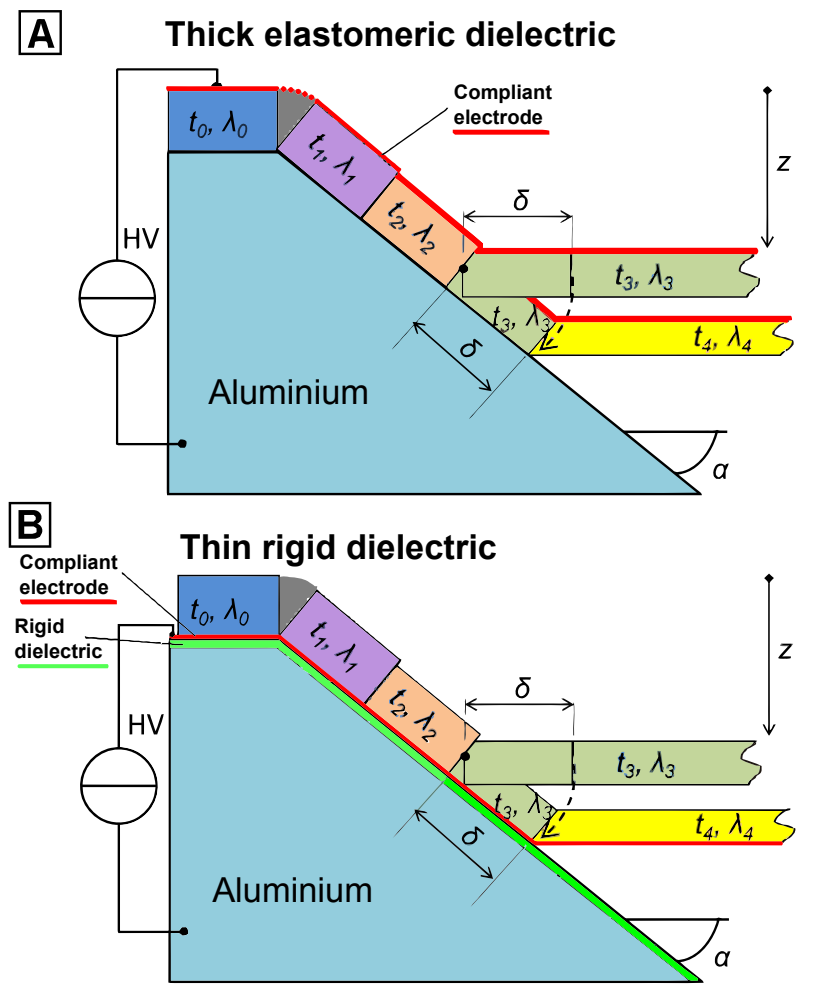

Figure 3. Cross-sections to illustrate the model behavior for the two zipping DEA structures. A Membrane dielectric model: the electric field builds up across the elastomer membrane. B Rigid dielectric model: the compliant electrode is patterned on the bottom of the membrane, and the electric field builds up across a thin high-quality rigid dielectric.

\subsection{Model hypotheses}

We pose a set of assumptions to implement the two models. The two dominant energy contributions are the electrostatic energy and the mechanical strain energy in the membrane. The bending energy and the surface energy (stiction) are neglected. The adhesive forces between the membrane and the rigid electrode would become apparent only in dynamic mode, when ramping down the voltage from a zipped state to a lower value of $z$. We neglect the fringing fields, which would bend down the central suspended part of the membrane. We assume that the membrane already in contact with the sidewalls is in a non-slipping condition, which implies that its thickness and stretch state varies over the zipping depth $z$. It is therefore necessary to implement an iterative process to compute the state of membrane elements. The flat suspended central part is considered to be under equibiaxial stretch. The polymer satisfies the volume incompressibility condition. Based on these hypotheses, we assume that the next zipped element of length $\delta$ comes from a rotation of the previous flat part (figure 3) and inherits its thickness and stretch state, as described previously [27].

\subsection{Procedure of the implemented algorithm}

The algorithm which outputs the vertical displacement in function of the applied voltage is structured as follows: 1) The voltage is set to $U=0$. 2) We compute the total energy function 
Table 1. Expression of the mechanical strain and electrostatic energies of the two models (not taking into account the sidewalls roughness).

\begin{tabular}{lll}
\hline Model & Mechanical strain energy & Electrostatic energy $E_{E S}=-\frac{1}{2} C U^{2}$ \\
\hline Thick elastomeric dielectric & $E_{M S}=W_{\text {flat }} V_{\text {flat }}+\sum_{i} W_{i}\left(\lambda_{i}\right) V_{i}$ & $E_{E S}=-\frac{1}{2} \epsilon_{0} \epsilon_{\text {mem }} \sum_{i}\left(\frac{A_{i}}{t_{i}}\right) U^{2}$ \\
Thin rigid dielectric & $E_{E S}=-\frac{1}{2} \epsilon_{0} \epsilon_{\text {diel }} \frac{A_{\text {lat }}(z)}{t_{\text {diel }}} U^{2}$ \\
\hline
\end{tabular}

in function of the zipping depth $E(z)$ with a resolution of $1 \mu m$ or below. 3) We look for the first minimum of $E(z)$, which will determine the stable vertical displacement $z$ that corresponds to the current voltage. 4) The voltage is incremented and we start again at step 2. We end the algorithm once the vertical displacement has reached the bottom of the chamber.

\subsection{Computation of the total energy function}

The mechanical strain energy is computed by multiplying the volume of each deflected membrane part by the elastic energy density $W$ of the Gent hyperelastic model in the equibiaxial case (equation 1). $\mu$ and $J$ are the Gent model parameters, and $\lambda_{1}=\lambda_{2}=\lambda$ is the equibiaxial linear stretch.

$$
W(\lambda)=\frac{\mu J}{2} \ln \left(1-\frac{2 \lambda^{2}+\lambda^{-4}-3}{J}\right)
$$

The expression of the mechanical strain and electrostatic energies for any deflection $z$ and voltage $U$ are expressed in the table $1, V$ being the volumes, $C$ the capacitance, $A$ the areas and $t$ the thicknesses. The indexes flat, mem, lat and diel stand for the central (flat) part, the membrane, the lateral (area), and the rigid dielectric.

The total energy is then:

$$
E(z)=E_{M S}(z)+E_{E S}(z)
$$

The first minimum of the function will determine the stable zipping depth. Examples of plotted energy curves can be found in our previous work [27]. It could also be possible to see the hysteresis (pull-out voltage) commonly observed in silicon zipping devices by finding the minimum starting from a zipped state (i.e. the highest value of $z$ ). However, the surface adhesion energy of the elastomer on the sidewall would add a non-negligible contribution that is difficult to estimate.

\subsection{Surface roughness of the sidewalls}

The analysis of the sidewalls profiles of our devices exhibit a significant roughness (average roughness $R_{a}$ and RMS roughness $R_{q}$ have been measured by white light interferometry in the range of 5 and $9 \mu \mathrm{m}$ respectively, see section 5.2). This rough surface may leave some air trapped between the membrane and the sidewalls. These air pockets act as a stacked series capacitor of lower dielectric constant and therefore reduce the electrostatic energy.

As a mean to evaluate the contribution of such an effect in the model, we can assume a saw tooth-shaped wall capacitance of peak-to-peak gap $R_{0}$, with a constant thickness elastomer membrane on top (figure 4). 


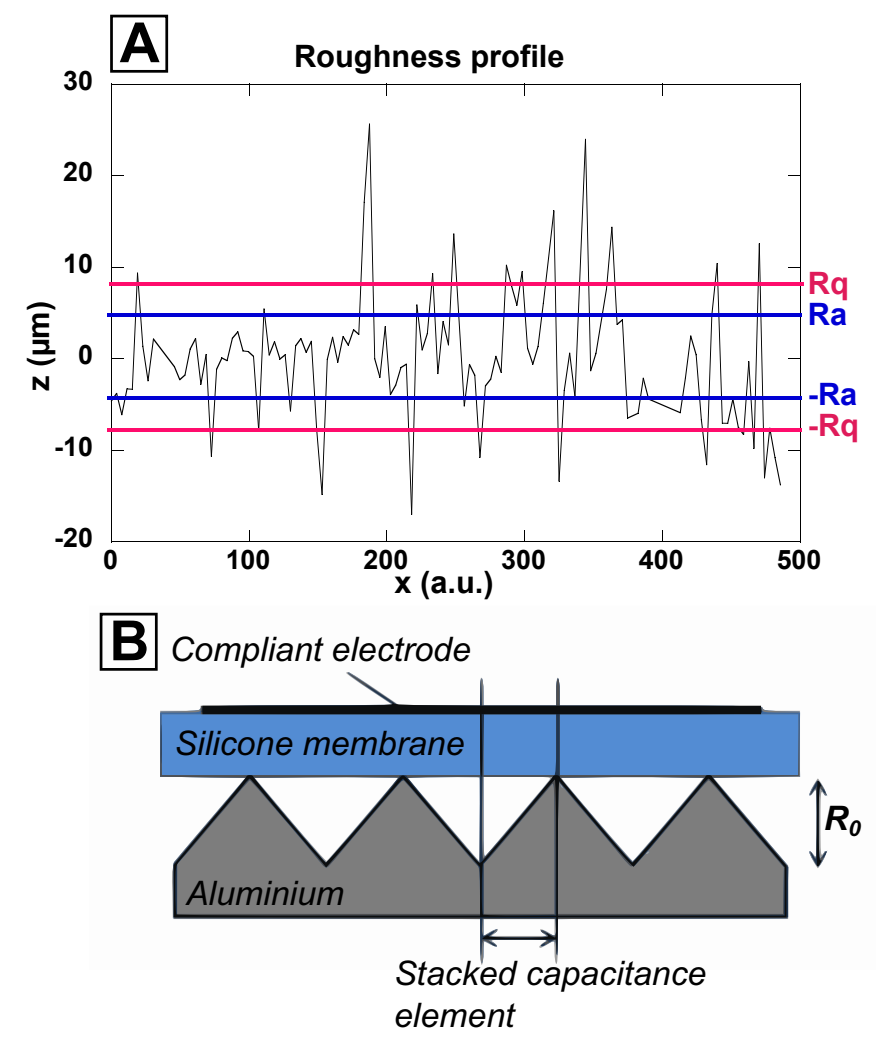

Figure 4. A Example of arbitrary typical roughness profile with statistical roughness parameters $R_{a}$ and $R_{q}$. B Roughness modeling. The model has a peak-to-peak roughness parameter $R_{0}$.

The capacitance $\mathrm{C}$ used in the electrostatic energy expression (table 1) corresponds to the smooth surface case and can be replaced by the rough surface case (equation 3 ).

$$
C_{\text {rough }}=\epsilon_{0} \frac{A}{R_{0}} \ln \left(\frac{\epsilon_{r, \text { mem }} R_{0}}{t_{\text {mem }}}+1\right)
$$

We shall use $R_{0}=2 \cdot R_{a}$ and $R_{0}=2 \cdot R_{q}$ as approximations.

\subsection{Electrical breakdown}

A main limitation of every DEA is the electrical breakdown, which defines the maximal achievable deflection. Dielectric breakdown of conventional DEAs (i.e. sandwiched between two compliant electrodes) occurs when the electric field in the dielectric exceeds the maximal value tolerated by the material. This failure mode may be triggered by the pull-in instability or wrinkling, or happen before these effects are present $[29,30]$. But the design of zipping DEAs requires paying attention to electrical failures triggered by actuation characteristics that are not present in squeezing-mode DEAs. The first of these characteristics is linked with the assumption of membrane non-slipping condition on the sidewalls. The highest electric field, and hence the point where dielectric breakdown will occur, is where the membrane is thinnest, i.e. on the outer periphery of the suspended part. Although the increasing stretch with $z$ enhances the breakdown field [9], we work in a range for which we do not expect a significant change.

Secondly, the deflection versus voltage characteristic of the zipping DEAs exhibits a very abrupt slope above a threshold voltage (onset of complete zipping, see figure 5). If the breakdown 
occurs during this zipping jump, the device fails, similarly to the well-known snap-in instability. Keplinger et al.s closed chamber volumes has been proposed to harness the snap-in instability, which resulted in giant voltage-triggered deformations [2]. Zipping DEAs offer even more design freedom to tune the bistable behavior of the actuators, since one can play with all the geometrical parameters of the cavity and with the membrane material. For instance, it is possible to avoid the breakdown during this zipping jump by mechanically limiting the maximal displacement of the membrane by truncating the chamber.

A last issue related to the electrical failure of zipping DEAs is the electrocreasing effect, which occurs when high electric fields are applied across elastomers bonded on a rigid substrate [31]; which is similar to the case of the membrane zipped on the sidewalls. The extrapolation of Wangs data for the polymer we use (Nusil CF19) would indicate a critical electric field in the range of 100 $\mathrm{V} / \mu \mathrm{m}$. Since it is close to the breakdown field of the elastomer, we cannot exclude the possibility of an electrocreasing-triggered breakdown.

\section{Model results and actuator design}

In figure 5, results of the thick elastomeric dielectric model have been plotted using the following parameters (defined on figure 2 and section 3.3): $D=2 \mathrm{~mm}, d=0.6 \mathrm{~mm}, \alpha=20^{\circ}, t_{0}=25 \mu \mathrm{m}$, $\lambda_{0}=1.1, \epsilon_{m e m}=3, R_{0}=0$ (no roughness), unless otherwise specified. The targeted application for our zipping actuators is a micropump. The main pump performance are the pressure and the flow rate, which depends on the displaced volume and period of an actuation cycle. Stiffer silicones provide less deflection and thus limit the stroke volume, but increase both the response time and the pressure exerted on the fluid. We therefore chose a relatively stiff elastomer, the CF19 from Nusil (1.2 MPa Youngs modulus). We fitted the Gent model parameters ( $\mu$ and $J$ ) of uniaxial pulltests data on CF19 elastomer stripes with and without electrodes and extracted an electrodes Youngs modulus of $1.93 \mathrm{MPa}$ using a bilayer approach (equation 4), with $Y$ and $T$ the Youngs modulus and relative thickness of the bilayer stack, membrane and electrode [32].

$$
Y_{b i l}=Y_{m e m} T_{m e m}+Y_{\text {elec }} T_{\text {elec }}
$$

Out of this formula, we can re-compute the Gent model parameter $\mu=Y / 3$ for any membrane thickness, keeping $J=80.4$ constant. The breakdown field of the elastomer was measured at 96 $V / \mu m$ in a non-stretched membrane, and is taken as constant in the model.

On figure 5A, one can clearly see that thanks to the high electrostatic energy stored in the thin rigid dielectric, this model zips more than $0.5 \mathrm{kV}$ earlier. On figure $5 \mathrm{~B}$, the effect of roughness is clear: it lowers the electrostatic energy at a given voltage, which shifts the zipping jump at higher voltages. Looking at figure $5 \mathrm{C}$, prestretching the membrane has a strong influence on the zipping voltage. In traditional DEAs, the prestretch improves drastically the maximal displacement by overcoming the pull-in instability, indirectly keeping the voltage low thanks to the thickness reduction. One cannot think in the same way for zipping DEAs, since the actuator structure is different (in hard contact with the sidewalls and without electric field en the central part, figure 2A). Low-prestretched zipping actuators work at lower voltages and have a more controllable zipping depth (smoother $z(V)$ slope), but the risk of the membrane remaining stuck to the chamber wall during the voltage ramp-down is increased. Highly-prestretched zipping actuators work at higher 

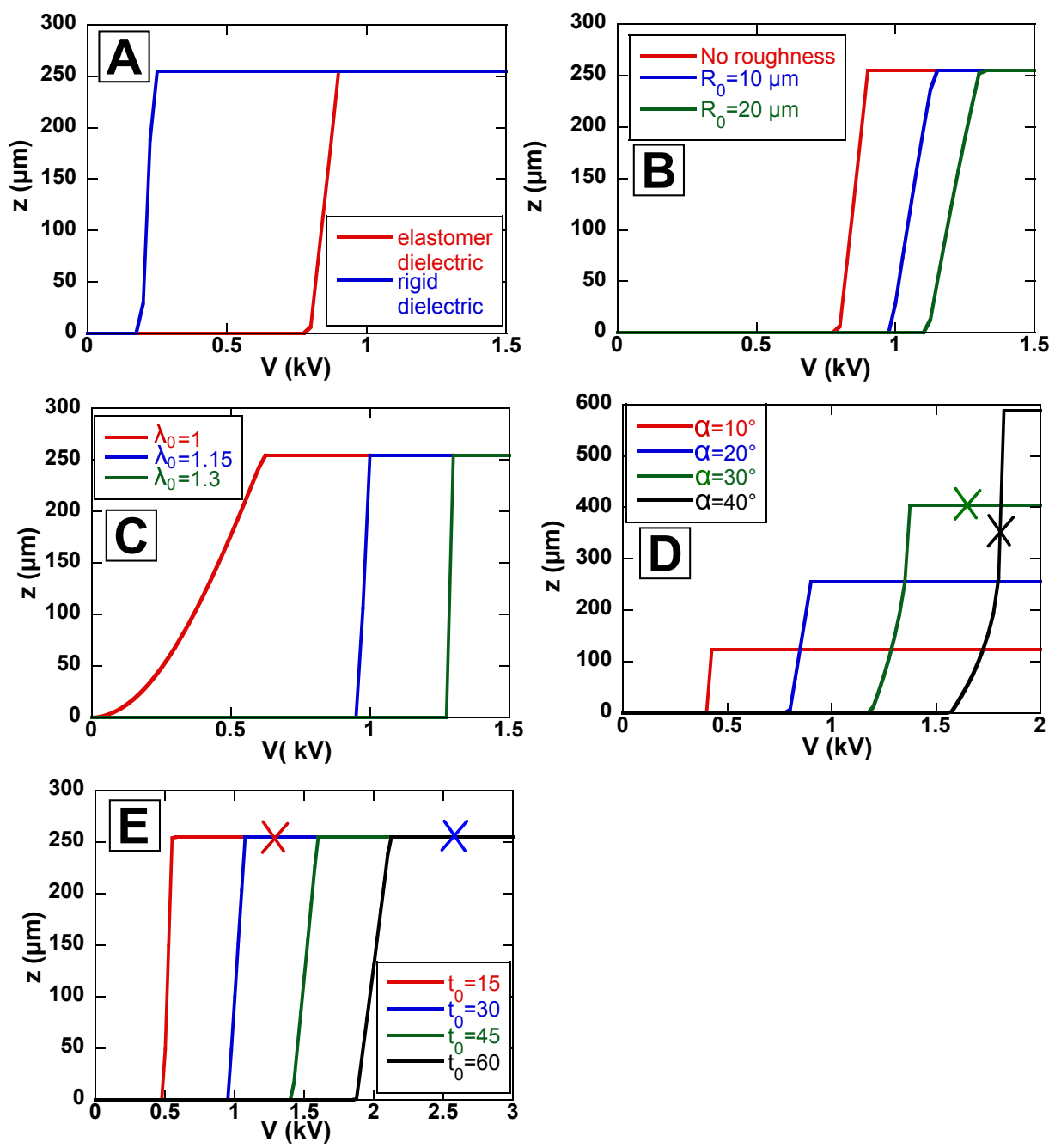

Figure 5. Results of the thick elastomeric dielectric model (except comparison of the 2 models on A). Main parameters (unless otherwise specified): $D=2 \mathrm{~mm}, d=0.6 \mathrm{~mm}, \alpha=20^{\circ}$, $t_{0}=25 \mu m, \lambda_{0}=1.1, \epsilon_{m e m}=3, R_{0}=0$ (no roughness). The breakdowns are indicated by crosses. A Comparison of the two models. The rigid dielectric has a thickness of $2 \mu m$ and a relative permittivity of $\epsilon_{\text {diel }}=3.12$. B Variation of roughness. C Variation of prestretch. D Variation of angle. E Variation of membrane thickness. The breakdown voltages for the 45 and $60 \mu \mathrm{m}$ membranes are at 3.8 and $5.1 \mathrm{kV}$.

voltages, exhibit a more pronounced bistable characteristic and have more mechanical energy for a given deflection, which counters membrane sticking and provide more force to move parts or fluids. The prestretch adds an energy barrier to the total energy curve and keeps the deflection at $z=0$ until being overcome by the electrostatic energy. Increasing the sidewalls angle (figure 5D) has a strong effect on both the zipping voltage and the maximal deflection (provided the chamber is not truncated). A smaller angle chamber zips at lower electric field, but provides less force and needs somewhat more prestretch to ensure a quick membrane detachment from the sidewalls. For a $40^{\circ}$ sidewalls chamber and the CF19 elastomer, we expect the breakdown to occur during the zipping jump. The figure 5E shows that the zipping jump scales almost linearly with the thickness, but one obtains more margin after the zipping jump before the breakdown occurs. 


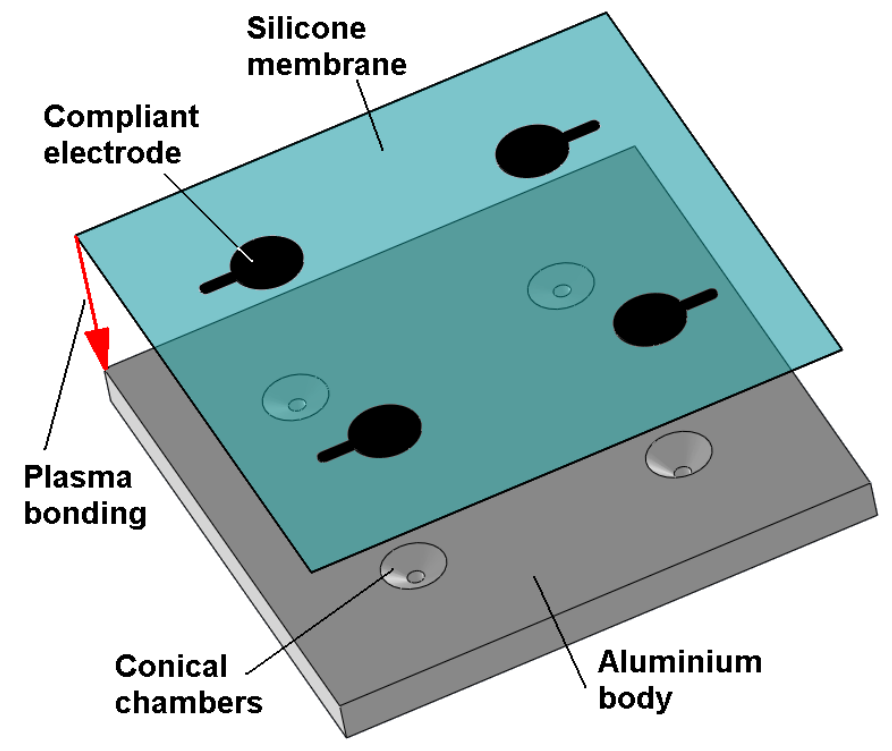

Figure 6. 3D view of the fabricated zipping devices. The compliant electrodes are patterned on top of the silicone membrane, and contacted with a PCB.

\section{Device fabrication}

A 3D view of the fabricated zipping devices is exposed on Figure 6. It consists in a silicone membrane with compliant electrodes patterned on top, bonded on an aluminium body that embeds machined conical chambers.

\subsection{Silicone membrane}

Many papers on DEA use acrylic elastomers such as VHB from 3M, which have the advantage being commercially available in films, and can be highly pre-stretched, enabling excellent static displacements. However, their high viscoelasticity, temperature sensitivity and reliability are disadvantages for commercial products. Silicones and polyurethanes seem to be the best choice candidates for reliable and fast actuators. We report below on our silicone membrane fabrication process, which gives us a maximal freedom regarding the choice of the elastomer and the thickness. Membranes for DEAs must be defect-free and present excellent thickness homogeneity in order to prevent premature breakdown. Casting a thin silicone membrane on a substrate involves the subsequent separation of the former from the latter. Pulling off the membrane induces a large deformation of the silicone layer, which must be avoided because of the Mullins effect [33]. We have consequently developed different methods based on the application of a silicone layer on a sacrificial thin-film for a facilitated release. We have used both spin-coating on silicon wafers and blade casting on glass plates, and we obtained better thickness homogeneity with the casting method, which also allows coating larger surfaces in a single run. Regarding sacrificial layers, we experimented with photoresist as well as water-soluble films such as poly(acrylic acid) (PAA), poly(vinyl alcohol) (PAA) and dextran, which have been successfully used for surface micromachining [34]. Photoresist must be dissolved in acetone, which swells the silicone membrane and can dissolve and wash away some silicone oil, leading to a modification of the mechanical 
properties [35]. Furthermore, the use of acetone is problematic on larger surfaces such as glass plates due to its high evaporation rate. We consequently favor the use of water-soluble sacrificial layers. Among dextran, PAA and PVA, we have obtained the best results with PAA in terms of ease of coating (wettability of the substrate, absence of pinholes) and resistance to the temperature used during the cross-linking.

The process is described on figure 7:

The first step is cleaning the glass plates. The surface cleanliness is very critical to obtain layers without defects. These preparation steps both removes all organic residues and lowers the surface tension of the glass. We use an adjustable gap ZUA2000 Zehnter applicator blade placed on a ZAA2300 automatic film applicator coater. A $30 \mu \mathrm{m}$ gap is set on the blade, and a $5 \%$ Poly(acrylic acid) (PAA) solution in water is casted on a heated glass plate (figure 7A). Once dried, this sacrificial layer is less than $2 \mu \mathrm{m}$ thick. The silicone is prepared by mixing the two components with isooctane or siloxane-based solvents to reduce its viscosity. The dried membrane thickness is lower than the blade gap, and empirical tests must be performed to be able to predict the desired value (figure 7B). Once casted, the silicone layer is left at ambient temperature to allow for the solvent to evaporate from the uncured layer, and then placed in an oven to complete the crosslinking (figure 7C). We then tape a laser-cutted flexible frame on top of the silicone layer (figure 7D). It prevents the fragile membrane from being stretched during the release. When using PAA, the glass plate is dipped into a hot water bath, and the flexible frame with the attached silicon membrane can be easily lifted from the plate (figure $7 \mathrm{E}$ ). The obtained membranes are $20 \mathrm{x} 30 \mathrm{~cm}^{2}$ silicone membrane sheets (limited by the applicator size) of 15 to more than $100 \mu \mathrm{m}$ thicknesses (targeted thickness typically reached within $\pm 3 \mu \mathrm{m}$ ), with a total thickness variation over the

whole sheet below $3 \mu \mathrm{m}$ ( $<1 \mu \mathrm{m}$ over a $5 \times 5 \mathrm{~cm}^{2}$ piece). We have successfully tested our coating process with several different silicones including Dow Corning Sylgard 186, Nusil CF19-2186, Nusil CF18-2186, Nusil R32-2186, and others. The most critical point is the amount of solvent needed to reach the good viscosity range, which can lead to the formation of voids in the membrane if the silicone crosslinks before it evaporates. The flexible frames have printed registration marks, so that the silicone membranes can be cut to any shape with very clean edges at a precision of $100 \mu \mathrm{m}$ by a Speedy300 laser with optical pattern recognition, from Trotec [36] (figure 7F). The clean laser cut avoids all defects that occur when cutting the membrane by mechanical means, which allows applying more prestretch before tearing. The stretch-free cutted membrane remains on a support ring by natural stiction, and is then transferred on the stretcher arms covered with double-sided tape (figure $7 \mathrm{G}$ ).

\subsection{Conical chamber}

Of the two zipping structures presented, one can see that if the electric field is applied across a thin rigid dielectric (figure $3 \mathrm{~B}$ ) such as $2 \mu \mathrm{m}$ of $\mathrm{SiO}$ or $\mathrm{SiN}$, the driving voltage is reduced. However, the realization of a pinhole-free rigid dielectric layer that is only of a few $\mu m$ thick is critical. We therefore chose to perform our experiments on zipping devices with the electric field applied across the elastomer membrane. The conical chambers were milled in an aluminium plate with a CNC from Step-Four basic series [37]. The conical tip of the drillbits was used to achieve the conical shape of the chambers, hence the set of angles $\alpha$ obtained of $15,22.5,31$ and $45^{\circ}$. The silicone 

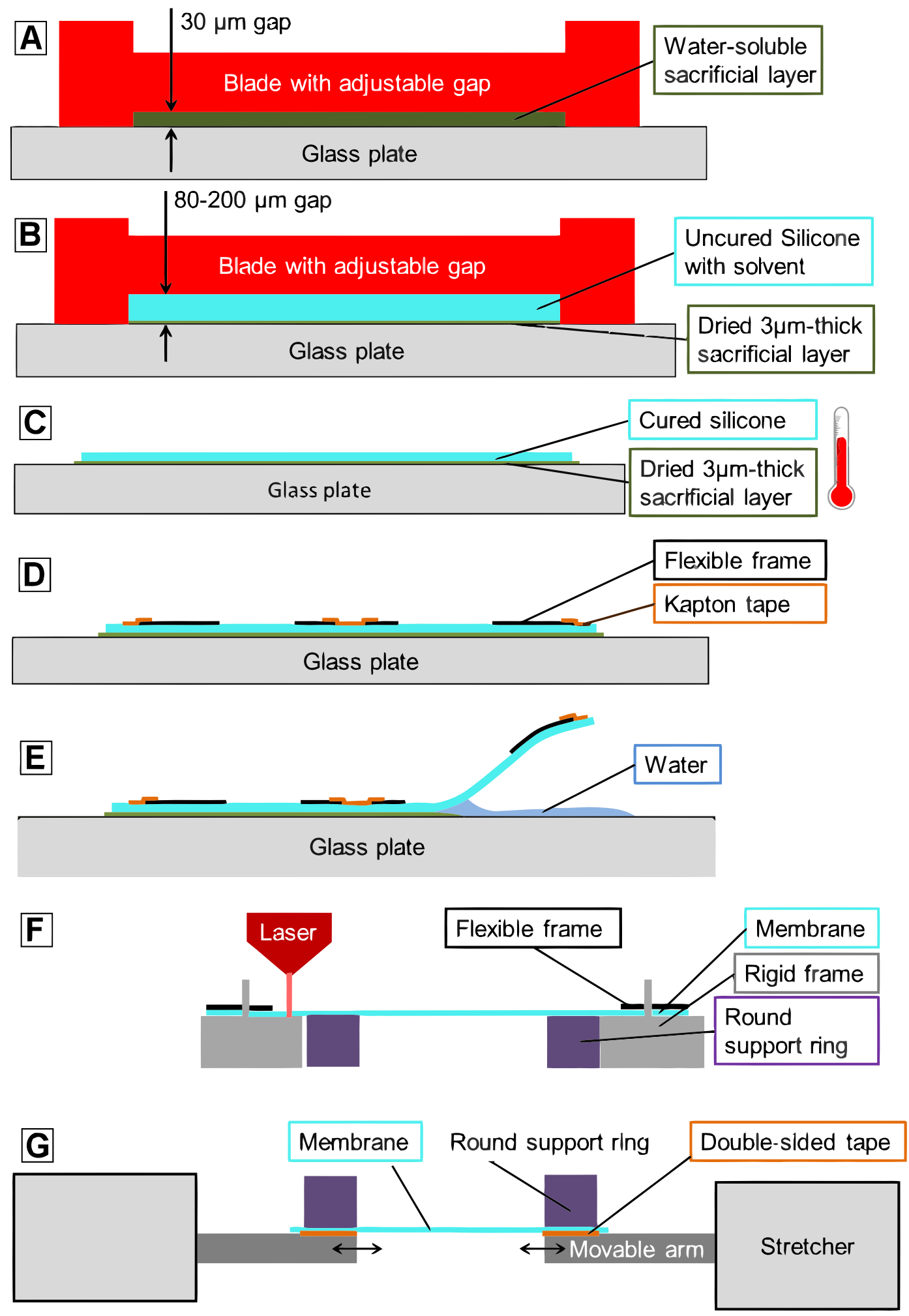

Figure 7. Membrane fabrication steps. A: Sacrificial layer casting. B: Silicone casting over the dried sacrificial layer. C: Silicone crosslinknig. D: Flexible frame taping. E: Release of the silicone membrane by dissolution of the sacrificial layer with water. F: Laser cutting. G: Prestretching. Photographs available online in the supplemental materials. 
Table 2. Dimensions of the measured actuators. $\lambda_{0}$ is the biaxial pre-stretch, and $\alpha$ the cone sidewalls angle

\begin{tabular}{ll|llll}
\hline \multicolumn{2}{c}{$\lambda_{0} \downarrow \alpha \rightarrow$} & $15^{\circ}$ & $22.5^{\circ}$ & $31^{\circ}$ & $45^{\circ}$ \\
\hline \multirow{2}{*}{1.13} & $D(m m)$ & 2.38 & 2.14 & 2.14 & 2.10 \\
& $t_{0}(\mu m)$ & 23.7 & 24.4 & 24.4 & 24.4 \\
\hline \multirow{2}{*}{1.27} & $D(m m)$ & 2.42 & 2.55 & & \\
& $t_{0}(\mu m)$ & 24.6 & 24.6 & & \\
\hline
\end{tabular}

membrane was pre-stretched to the desired value using a home-made stretcher with eight movable fingers. We compute the equibiaxial prestretch using the ratio of the measured thicknesses before and after prestretch.

\subsection{Electrode patterning and membrane bonding}

A compliant electrode composed of 1:10wt of carbon black and a soft silicone was printed on top of the membrane by an indirect stamping technique (pad printing). These polymer-carbon composite electrodes have the advantages of being well bonded to the membrane, but are stiffer than if dispersed in grease. It is therefore needed to keep them thin relative to the membrane thickness to reduce the stiffening impact [38]. We obtained electrode thicknesses between 1 and 3.5 $\mu m$, which increases the Youngs modulus of a $20 \mu m$ membrane by less than $10 \%$. We irreversibly bond the printed membrane onto the aluminum chambers by activating the silicone and the native oxide with an oxygen plasma. Over the conical holes, the membrane is suspended and the surface activation of the silicone decays within a few hours so that it has no influence when actuating the device at a later stage. The devices were clamped to a PCB to which electrical contact was made using a conducting varnish.

\section{Experimental results}

The profile of the zipped membranes were measured by automated frame stitching on a white light interferometer Wyko NT1100 DMEMS from Veeco. The top diameters and membrane thickness of the 6 measured actuators can be found in table 2. The bottom diameters $d$ are comprised between 0.6 and $1 \mathrm{~mm}$.

\subsection{Zipping behavior}

As explained previously, unless the sidewalls angle is large (54 in our earlier work [27]), zipping DEAs need to be prestretched to provide the restoring force to counter the adhesion forces between the aluminium and the silicone, at a cost of a higher driving voltage. This effect is difficult to predict theoretically, and we therefore prestretched equibiaxially two set of CF19 membranes with $\lambda_{0}=1.13 \pm 0.01$ and $\lambda_{0}=1.27 \pm 0.01$. All membranes were able to come back in their initial position within a few seconds of turning off the voltage, which means that a prestretch of 1.13 or lower is enough for our devices. 

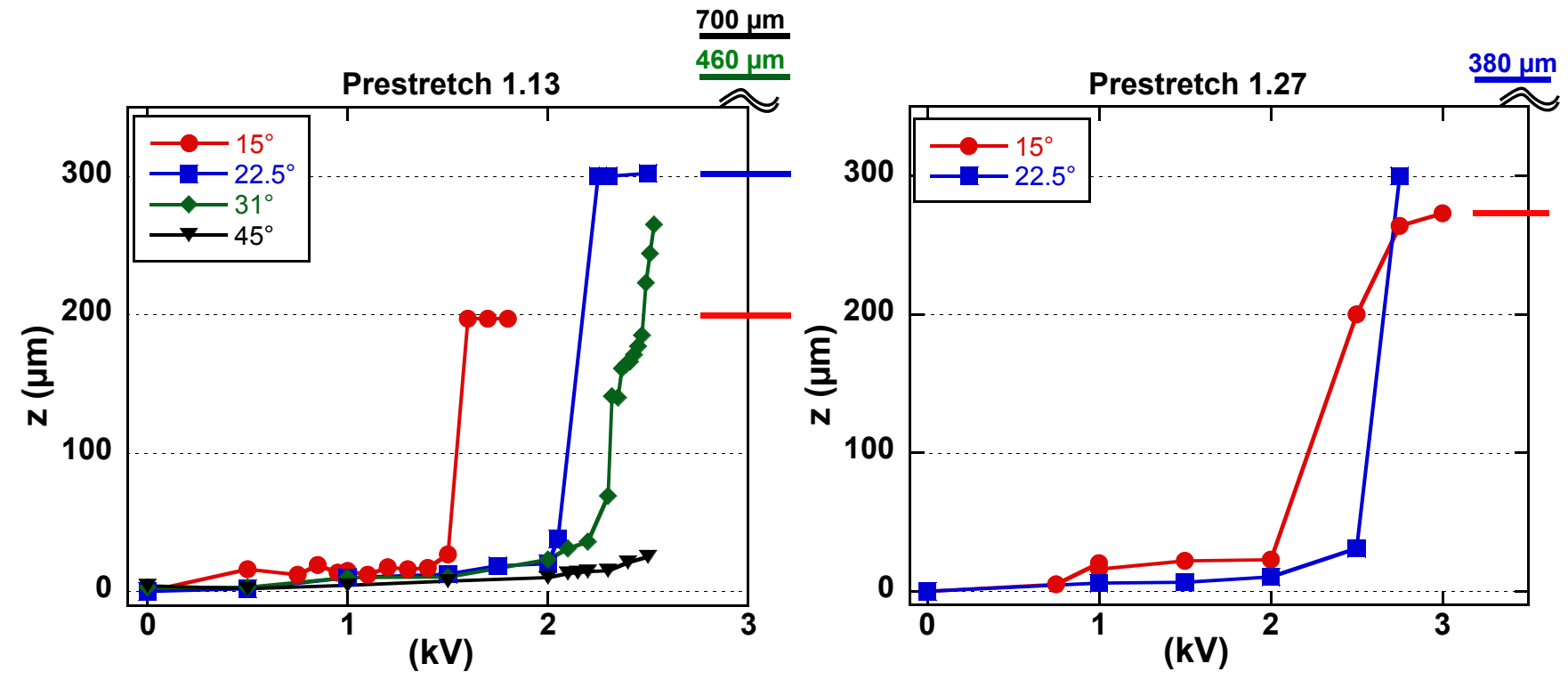

Figure 8. Vertical deflection of the zipped membranes. The maximal deflections (limited by the bottom of the chambers) are indicated by the bold lines at the right of the plot. The lines are guides to the eye.

The figure 8 represents the zipping curves of the measured samples. The $15^{\circ}$ and $22.5^{\circ}$ lowprestretched chambers could zip down to the bottom of the chamber, but only the $15^{\circ}$ one on the set of high-prestretched ones.

\subsection{Shape of deflected membrane}

The membrane profiles during the zipping jump have been plotted on the figure 9 , together with the aluminium sidewalls. The cross-section profile data have been shifted up by the membrane thickness. Since each measurement is processed independently, a small offset of the cross-section can result in a slightly different diameter size, which may explains that the datapoints of the membrane profile are very close to the sidewalls on top of the device.

\section{Comparison of the results with the model}

\subsection{Shape of the deflected membrane}

As seen on figure 9, the measured shape of the membrane is very close to the one we assumed in the model: completely in contact with the sidewalls, and a flat part in the center. However, one can notice that at $2.3 \mathrm{kV}$, the membrane is clearly not yet in contact with the sidewalls, but is bending down because of the electric field. This effect is visible on the $z(V)$ plots (figure 8, for which the deflection is a few tens of $\mu \mathrm{m}$ before starting to zip. Secondly, it seems that the membrane is visibly thinner in the top of the cavity $(0<z<100 \mu \mathrm{m})$, and somewhat thicker below $(100<z<200 \mu \mathrm{m})$. This might possibly be a discrepancy coming from the automated stitching measurements, since there are less datapoints and more noise on the sidewalls. More likely, the membrane experiences a compression and/or sliding on the sidewalls; maybe favored 


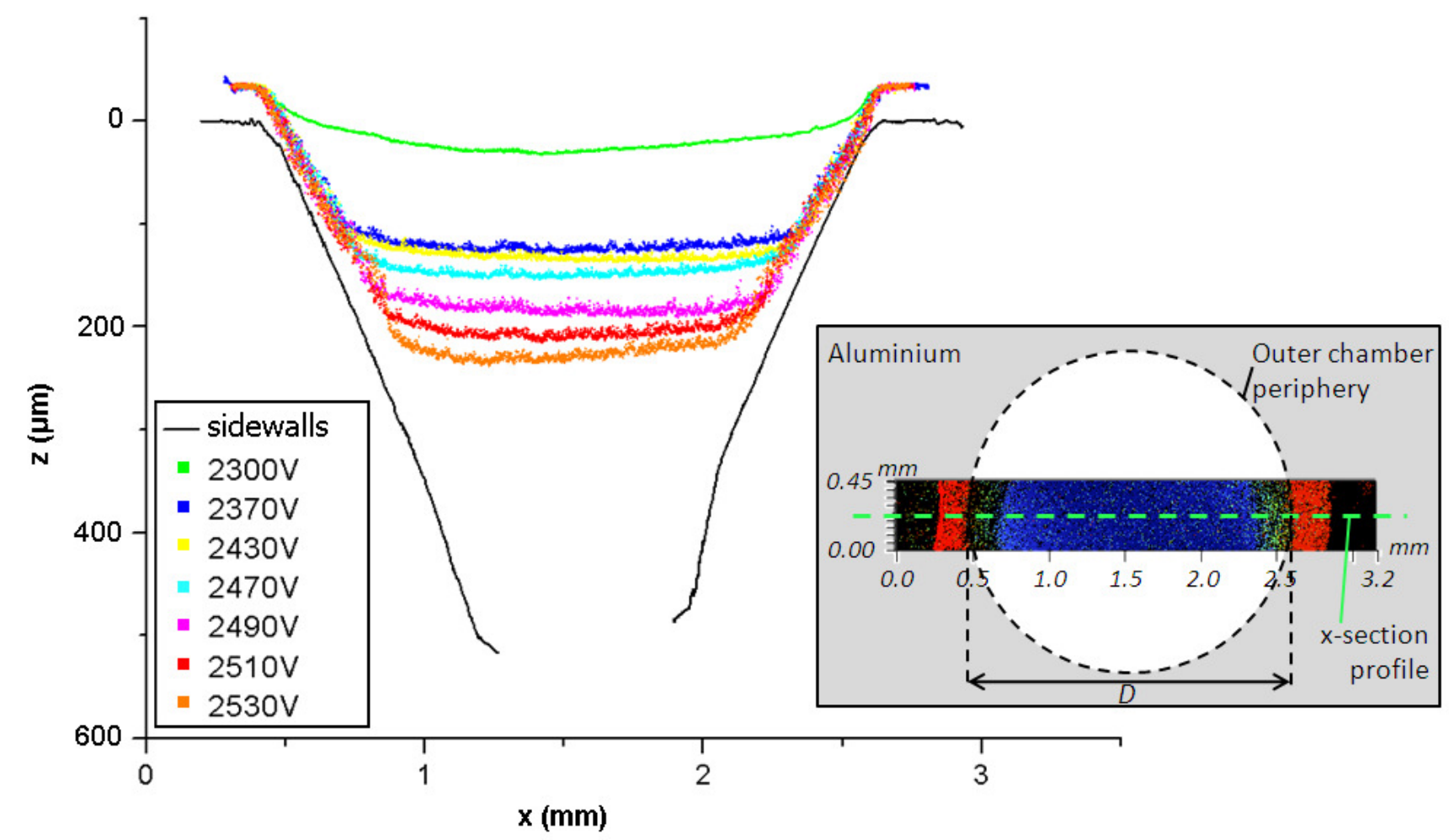

Figure 9. Membrane profile of the device with $\lambda_{0}=1.12$ and $\alpha=31^{\circ}$. (x and y axes not at the same scale). The insert shows a $3 \mathrm{D}$ dataset from the white light interferometer, from which the cross-section profiles have been extracted.

by the high roughness. One other reason could be that the first $100 \mu \mathrm{m}$ are zipped at once; the membrane being in the same time bulging inside the cavity and pulled sideward in contact with the walls (from 2.3 to $2.37 \mathrm{kV}$ ). If one looks at the corresponding data of the $31^{\circ}$ chamber of figure 8 , a jump is indeed visible in the first part of the zipping curve. For $z>100 \mu m$, the zipping would proceed in continuous fashion.

\subsection{Zipping voltages}

We compare measured and predicted zipping voltages $V_{z i p}$, where $V_{z i p}$ is defined as the voltage in the middle of the steep zipping slope (figure 8), taking the average between the voltages of the start of zipping and when the membrane is fully zipped. For a few devices which had a dielectric breakdown just before reaching the bottom of the chamber, the zipping voltage is computed using a linear approximation, since we know the maximal zipping depth.

The figure 10 presents the measured zipping voltages at both low and high prestretch (in red) together with the zipping voltages from the model. Since every device has small variations of diameters and membrane thickness, each point from the model is computed using the exact measured values of the device. Two roughness values $R_{0}$ of 10 and $20 \mu \mathrm{m}$ are shown together with the case without roughness. According to our surface measurements, a value of $R_{0}=10 \mu \mathrm{m}$ seems reasonable and a value of $20 \mu m$ the upper limit.

While the predicted voltages are roughly $1 \mathrm{kV}$ lower than the measured one, the model 

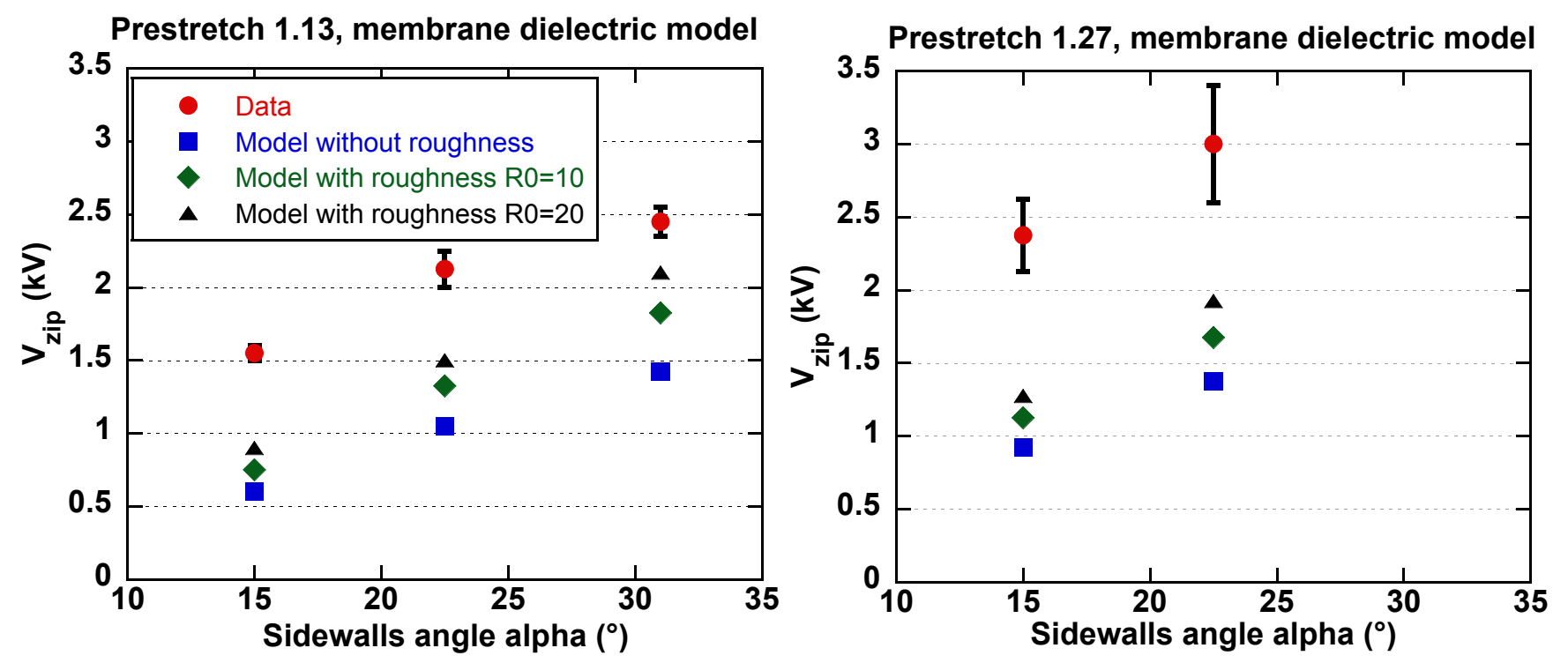

Figure 10. Zipping voltages of the measurements and the model without sidewalls roughness and with a peak-to-peak model roughness of 10 and $20 \mu \mathrm{m}$.

accurately predicts the effect of changing the sidewalls angle on the zipping voltage, the difference between the measured and predicted zipping voltage being constant for a same prestretch. With the assumption of a $10 \mu \mathrm{m}$ roughness, the difference is in the range of $0.7 \mathrm{kV}$ at low prestretch and $1.3 \mathrm{kV}$ at high prestretch. This difference indicates that our model is more accurate at lower prestretches. In previous work, we indeed measured a good agreement between the model and the data in pyramidal-shape chambers which were not using any prestretch to release the membrane thanks to the large angle $\left(54.7^{\circ}\right)[27]$.

The effect of surface roughness cannot explain the full discrepancy. Even with the hypothesis of $R_{0}=20 \mu \mathrm{m}$, the difference between the predicted and measured zipping voltages remains too large. The larger difference of the highly prestretched devices compared to the lower prestretched ones (figure 10) could possibly reflect that if the membrane is more prestretched, it conforms less inside the valleys of the rough surface and leaves larger air gaps.

The most likely explanation for the larger measured than simulated $V_{z i p}$ is because our model neglects the membrane bending energy. The maximum of the mechanical stretching energy ranges from a few $\mu J$ to $20 \mu J$ depending on the actuators parameters. The bending energy needed to bend from a flat position into the zipped shape (figure 2 and figure 9 ) is in the range of 0.3 to $1 \mu \mathrm{J}$. Also, the bending energy increases in the first tens of $\mu m$ of zipping and is then nearly independent of zipping depth. Including the bending energy would thus lead to an increase in predicted $V_{z i p}$, as it effectively adds an energy barrier that must be overcome to initiate zipping. The bending energy could also be the explanation of the smaller difference between theoretical and experimental $V_{z i p}$ of the low compared to the highly prestretched actuators (figure 10), since it is proportional to the effective Youngs modulus which increases with the prestretch. 


\section{Zipping DEA as a new class of actuators offering unique characteristics}

Zipping DEAs are a new type of DEA actuators which open new design spaces and could fit the needs of many applications. It allows out-of plane motion with high force, and is best suited for mm-size devices, from micropumps to tunable lenses to Braille displays. The deflected shape is very well controlled as it is given by the shape of the frame, which can be conventionally machined, molded or micromachined [27]. The voltage vs. displacement characteristic can be tuned to virtually any shape by changing the sidewalls profile. The bistable behavior observed on some conventional DEAs relies on the softening and then hyperelastic stiffening of the stressstrain characteristic of the elastomer materials [2]. If one wants to operate in bistable mode, a careful choice of the elastomer and operating conditions (such as pre-stretch or offset pressure) is necessary, and imposes strong design restrictions. In the case of zipping DEAs, the presence and/or threshold voltage of one or several bistable jump(s) can be chosen by playing not only with the elastomer and the prestretch state, but also with the chambers geometry. This opens the door to a fine tuning of bistable or multi-stable modes, for applications like braille displays or step-bystep positioning systems. By including specific features like embedded channels or holes in the chamber [27], one also benefits from the electrostatic pressure of the membrane on the sidewalls to hermetically seal these apertures, building for instance effective pumping and valving devices for integrated microfluidics or gas control. As also pointed out in previous work [27], variants of the measured zipping DEA structure are also possible. Instead of being applied across the membrane, the electric field can also build up across a solid layer deposited on the rigid electrode or a stack of both [26, 28,39]. Hybrid actuators including squeezing and zipping modes might also be of particular interest. We could expect important voltage reductions if the electric field is applied across a rigid dielectric. These devices would need a few $\mu m$-thick high quality rigid dielectric to be coated or grown on the rigid electrode, and the compliant electrode is patterned on the lower side of the membrane so that it comes in contact with the dielectric. If the enhancement of relative permittivity and foremost breakdown voltage would theoretically drastically lower the actuation voltage, it is practically difficult to fabricate a homogeneous and pinhole-free rigid dielectric. The well-established microfabrication technologies would be well suited to build such zipping DEA structures, but the critical would then be the realization of the sloped chamber sidewalls, since most of the microfabrication processes are designed to yield vertical etching.

\section{Conclusion}

We studied theoretically and experimentally zipping DEAs. We developed two analytical models corresponding to two zipping DEA structures, the electric field being either applied across the elastomer membrane or across a rigid dielectric. Our models take as input a wide range of parameters (actuator geometry, sidewalls roughness, dielectric properties and membrane hyperelastic behavior), so that any configuration can be quickly evaluated. We experimentally validated the membrane dielectric model, showing that the effect of sidewalls angle on the zipping voltage behaves as predicted by our model, although a systematic shift of the zipping voltage was observed on all samples. This shift is in the range of $1 \mathrm{kV}$ for the devices prestretched to 1.23 and of $1.5 \mathrm{kV}$ with a prestretch of 1.27 , and is attributed to the bending energy which was not 
included in the model. We successfully demonstrated that it is possible to achieve a bistable full zipping in $2.3 \mathrm{~mm}$ diameter conical chambers with $15^{\circ}$ and $22.5^{\circ}$ sidewalls angle, which fits the requirements for large-scale integrated microfluidic actuators (MLSI). MLSI devices powered by $m m$-size DEAs will be truly portable, avoiding the use of large pneumatic off-chip equipment. It would for instance suit the point-of care detection of low concentration molecules in large $(\mu L$ or above) sample volumes, such as for HIV viral load [40].

\section{Acknowledgements}

The authors greatly acknowledge Ms. Samin Akbari, Mr. Jun Shintake, Dr. Seun Araromi and Mr. Alexandre Poulin for their precious collaboration. This work was funded by the Swiss National Science Foundation grant 200020-140394.

\section{References}

[1] Ron Pelrine, Roy Kornbluh, Qibing Pei, and Jose Joseph. High-speed electrically actuated elastomers with strain greater than 100\%. Science, 287:836-839, 2000.

[2] Christoph Keplinger, Tiefeng Li, Richard Baumgartner, Zhigang Suo, and Siegfried Bauer. Harnessing snapthrough instability in soft dielectrics to achieve giant voltage-triggered deformation. Soft Matter, 8:285-288, 2012.

[3] Roy Kornbluh, Ron Pelrine, Qibing Pei, Seajin Oh, and Jose Joseph. Ultrahigh strain response of field-actuated elastomeric polymers. In Proc. SPIE, volume 3987, pages 51-64, 2000.

[4] Iain A. Anderson, Todd A Gisby, Thomas G McKay, Benjamin M. O'Brien, and Emilio P. Calius. Multifunctional dielectric elastomer artificial muscles for soft and smart machines. Journal Of Applied Physics, 112:04110-041101-20, 2012.

[5] C. Jordi, S. Michel, and E. Fink. Fish-like propulsion of an airship with planar membrane dielectric elastomer actuators. Bioinspiration and Biomimetics, 5:1-9, 2010.

[6] www.vivitouch.com.

[7] Federico Carpi, Gabriele Frediani, Simona Turco, and Danilo De Rossi. Bioinspired tunable lens with musclelike electroactive elastomers. Advanced Functional Materials, 21:4152-4158, 2011.

[8] Takehiro Sugimoto, Kazuho Ono, and Akio Ando. Semicylindrical acoustic transducer from a dielectric elastomer film with compliant electrodes. Journal of the Acoustical Society of America, 130:744-752, 2011.

[9] Samin Akbari and Herbert R. Shea. An array of 100um x 100um dielectric elastomer actuators with $80 \%$ strain for tissue engineering applications. Sensors and Actuators, A: Physical, 186:236-241, 2012.

[10] Soo Jin Adrian Koh, Christoph Keplinger, Tiefeng Li, Siegfried Bauer, and Zhigang Suo. Dielectric elastomer generators: How much energy can be converted? IEEE/ASME Transactions on Mechatronics, 16(1):33-41, 2011.

[11] Roy D. Kornbluh, Ron Pelrine, Harsha Prahlad, Annjoe Wong-Foy, Brian McCoy, Susan Kim, Joseph Eckerle, and Tom Low. From boots to buoys: Promises and challenges of dielectric elastomer energy harvesting. In Proc. SPIE, volume 7976, pages 1-19, 2011.

[12] Benjamin M. O'Brien, Emilio P. Calius, Tokushu Inamura, Sheng Q. Xie, and Iain A. Anderson. Dielectric elastomer switches for smart artificial muscles. Applied Physics A: Materials Science \& Processing, 100:385$389,2010$.

[13] Luis M. Fidalgo and Sebastian J. Maerkl. A software-programmable microfluidic device for automated biology. Lab on a Chip, 11:1612-1619, 2011.

[14] Erik. C. Jensen, Amanda M. Stockton, Thomas N. Chiesl, Jungkyu Kim, Abhisek Bera, and Richard A. Mathies. Digitally programmable microfluidic automation for multiscale combinatorial mixing and sample processing. Lab on Chip, 13:288-296, 2013. 
[15] Marc A. Unger, Hou-Pu Chou, Todd Thorsen, Axel Scherer, and Stephen R. Quake. Monolithic microfabricated valves and pumps by multilayer soft lithography. Science, 288:113-116, 2000.

[16] Meng-Ping Chang and Michel M. Maharbiz. Electrostatically-driven elastomer components for userreconfigurable high density microfluidics. Lab on a Chip, 9:1274-1281, 2009.

[17] Ronald E. Pelrine, Roy D. Kornbluh, and Jose P. Joseph. Electrostriction of polymer dielectrics with compliant electrodes as a means of actuation. Sensors and Actuators A: Physical, 64(1):77 - 85, 1998. Tenth IEEE International Workshop on Micro Electro Mechanical Systems.

[18] Roy Kornbluh. Dielectric Elastomers as Electromechanical Transducers, chapter 8: Fundamental Configurations for Dielectric Elastomer Actuators, pages 79-90. Elsevier, 2008.

[19] A. Wingert, M.D. Lichter, and S. Dubowsky. On the design of large degree-of-freedom digital mechatronic devices based on bistable dielectric elastomer actuators. IEEE/ASME Transactions on Mechatronics, 11(4):448-456, 2006.

[20] G. Kofod, W. Wirges, M. Paajanen, and S. Bauer. Energy minimization for self-organized structure formation and actuation. Applied Physics Letters, 90(8), 2007.

[21] S. Rosset, M. Niklaus, P. Dubois, and H.R. Shea. Large-stroke dielectric elastomer actuators with ion-implanted electrodes. Journal of Microelectromechanical Systems, 18(6):1300-1308, 2009.

[22] F. Carpi, G. Frediani, and D. De Rossi. Hydrostatically coupled dielectric elastomer actuators. IEEE/ASME Transactions on Mechatronics, 15(2):308-315, 2010.

[23] F. Carpi, C. Salaris, and D. De Rossi. Folded dielectric elastomer actuators. Smart Materials and Structures, 16(2):S300-S305, 2007.

[24] G. Kovacs, L. Dring, S. Michel, and G. Terrasi. Stacked dielectric elastomer actuator for tensile force transmission. Sensors and Actuators, A: Physical, 155(2):299-307, 2009.

[25] Jens Branebjerg and Peter Gravesen. A new electrostatic actuator providing improved stroke length and force. In Proceedings of the IEEE Micro Electro Mechanical Systems Workshop, pages 6-11, 1992.

[26] Pit Gebbers, Chauncey Graetzel, Luc Maffli, Christoph Stamm, and Herbert R. Shea. Zipping it up: Deas independent of the elastomer's electric breakdown field. In Proc. SPIE, volume 8340, pages 2P-1-2P-14, 2012.

[27] Luc Maffli, Benjamin M. O'Brien, Samuel Rosset, and Herbert R. Shea. Pump it up. In Proc. SPIE, volume 8340, pages 2Q1-2Q16, 2012.

[28] M.T.A. Saif, B.E. Alaca, and H. Sehitoglu. Analytical modeling of electrostatic membrane actuator for micro pumps. Journal of Microelectromechanical Systems, 8(3):335-345, 1999.

[29] S.J.A. Koh, T. Li, J. Zhou, X. Zhao, W. Hong, J. Zhu, and Z. Suo. Mechanisms of large actuation strain in dielectric elastomers. Journal of Polymer Science, Part B: Polymer Physics, 49(7):504-515, 2011.

[30] J.-S. Plante and S. Dubowsky. Large-scale failure modes of dielectric elastomer actuators. International Journal of Solids and Structures, 43(25-26):7727-7751, 2006.

[31] Q. Wang, L. Zhang, and X. Zhao. Creasing to cratering instability in polymers under ultrahigh electric fields. Physical Review Letters, 106(11):118301, 1-4, 2011.

[32] I. M. Ward. The Mechanical Properties of Solid Polymers. Chichester, 2004.

[33] L. Mullins. Softening of rubber by deformation. Rubber Chemistry and Technology, 42(1):339-362, 1969.

[34] V. Linder, B.D. Gates, D. Ryan, B.A. Parviz, and G.M. Whitesides. Water-soluble sacrificial layers for surface micromachining. Small, 1(7):730-736, 2005.

[35] K. Flittner, M. Schlosser, and H. F. Schlaak. Influence of solvents on the mechanical properties of dielectric elastomer actuators. In EuroEAP 2012 International conference on EAP transducers and artificial muscles, 2012.

[36] www.troteclaser.com.

[37] www.step-four.at.

[38] S. Rosset and H.R. Shea. Flexible and stretchable electrodes for dielectric elastomer actuators. Applied Physics A: Materials Science and Processing, pages 1-27, 2012.

[39] J. Han and M.A. Shannon. Smooth contact capacitive pressure sensors in touch- and peeling-mode operation. IEEE Sensors Journal, 9(3):199-206, 2009.

[40] T. Bourlet, A. Signori-Schmuck, L. Roche, V. Icard, H. Saoudin, M.-A. Trabaud, J.-C. Tardy, P. Morand, B. Pozzetto, R. Ecochard, and P. Andr. Hiv-1 load comparison using four commercial real-time assays. 
Journal of Clinical Microbiology, 49(1):292-297, 2011. 